\title{
Quantum cryptography on noisy channels: quantum versus classical key-agreement protocols
}

\author{
N. Gisin ${ }^{(1)}$ and S. Wolf ${ }^{(2)}$ \\ (1) Group of Applied Physics, University of Geneva, 1211 Geneva, Switzerland \\ (2) Dept. of Computer Science, Swiss Federal Institute of Technology (ETH Zürich), ETH Zentrum, 8092 Zurich, Switzerland
}

(November 14, 2018)

When the 4-state or the 6-state protocol of quantum cryptography is carried out on a noisy (i.e. realistic) quantum channel, then the raw key has to be processed to reduce the information of an adversary Eve down to an arbitrarily low value, providing Alice and Bob with a secret key. In principle, quantum algorithms as well as classical algorithms can be used for this processing. A natural question is: up to which error rate on the raw key is a secret-key agreement at all possible? Under the assumption of incoherent eavesdropping, we find that the quantum and classical limits are precisely the same: as long as Alice and Bob share some entanglement both quantum and classical protocols provide secret keys.

Quantum cryptography lies at the intersection of two of the major sciences of the 20th century: quantum mechanics and information theory. Moreover, due to the intimate relation between quantum cryptography and quantum non-locality, another major scientific achievement of this century, relativity, is not far away. This letter concerns the dialog between quantum physics and information theory in the context of optimal eavesdropping on a quantum channel and the corresponding secret-key agreement that, in principle, both quantum and classical algorithms provide. This analysis of the interplay of these complementary approaches reveals surprising connections.

To provide a secure communication, quantum cryptography [1] exploits quantum correlations to establish secure keys (which are then the basis for standard information-based cryptosystems). Alice prepares a pair of qubits (i.e. a pair of spin $\frac{1}{2}$ ) in a maximally entangled state, sends one qubit to Bob and keeps the other one. Then they both measure their qubit in a basis chosen independently at random within a set of 2 or 3 bases for the 4- and 6-state protocols, respectively. (Alice could also prepare a qubit in a state compatible with one of the bases and send it to Bob, but the protocol with qubit pairs is equivalent and better suited for the purpose of this letter). On a perfect channel the correlations are maximal and the protocol is straightforward, the secure key results essentially without any information theoretical algorithm. However, in practice the quantum channel is noisy and elaborated protocols are needed. These require as input an upper bound on the information accessible to the eavesdropper, a bound set by the laws of quantum physics. Natural questions are: up to which error rate is secure key agreement at all possible? This letter presents the answer to this question under the assumption of incoherent eavesdropping.

Below, we first review general incoherent eavesdropping and summarize recent results on secret-key agreement by public discussion. Then, the cases that Bob has more or less information than Eve are treated.

By general incoherent eavesdropping we mean the following. First, Eve lets each qubit sent by Alice to Bob interact with independent ancillas. The dimension of the ancillas and the interaction are arbitrary, except that the interaction is described by a unitary operator $U$ :

$$
\begin{aligned}
& U|\uparrow\rangle \otimes|0\rangle=\sqrt{\mathcal{F}} \uparrow\rangle \otimes \psi_{\uparrow}+\sqrt{\mathcal{D}}|\downarrow\rangle \otimes \phi_{\uparrow} \\
& U|\downarrow\rangle \otimes|0\rangle=\sqrt{\mathcal{F}}|\downarrow\rangle \otimes \psi_{\downarrow}+\sqrt{\mathcal{D}}|\uparrow\rangle \otimes \phi_{\downarrow}
\end{aligned}
$$

where the $\psi_{j}$ and $\phi_{j}$ are the normalized states of Eve's ancilla when Bob receives the qubit undisturbed and disturbed, respectively. The former case happens with probability $\mathcal{F}$, called the fidelity, and the latter with probability $\mathcal{D}$, called the disturbance or, equivalently, the Quantum Bit Error Rate (QBER). Next, Eve stores her ancillas until she learns the bases used by Alice to encode the qubits. Finally, she measures her ancillas one after the other, using any measurement scheme compatible with the laws of quantum physics. Clearly, if Eve interacts only weakly with the qubits, then she disturbs the channel only weakly, hence the QBER is low, but Eve gets little information. On the contrary, if the interaction is strong, Eve gains more information, but the QBER is larger. Optimal here means that for any given QBER, Eve chooses the qubit-ancilla interaction and her measurement to maximize her information. The QBER can be well estimated by Alice and Bob by comparing a fraction of their (classical) bits. From this and from the laws of quantum physics they can bound Eve's information.

The state vectors $\psi_{j}$ and $\phi_{j}$ in (11, 2) have to be such that they define a unitary operator $U$. Moreover, we choose them such that $U$ has the same effect on all qubits sent by Alice (all the Poincaré vectors are shrunk by the same factor). This is called symmetric eavesdropping. It simplifies the analysis considerably. It is important to note that one can assume symmetric eavesdropping without loss of generality, as proven in [2, 3]. Indeed, Eve can make her strategy look symmetric to Bob by applying arbitrary rotations $R$ to the qubit immediately before and $R^{-1}$ immediately after it interacts with her 
ancilla. As she knows which rotation she applies, she does not lose information. From Bob's point of view, however, the arbitrary rotations make the disturbance appear symmetric. From this symmetry condition one obtains the following relation for the fidelity $\mathcal{F}$ [2

$$
\mathcal{F}=\frac{1+\left\langle\phi_{\uparrow} \mid \phi_{\downarrow}\right\rangle}{2-\left\langle\psi_{\uparrow} \mid \psi_{\downarrow}\right\rangle+\left\langle\phi_{\uparrow} \mid \phi_{\downarrow}\right\rangle}
$$

The explicit form of the $\psi_{j}$ and $\phi_{j}$ and Eve's optimal measurements are given in [2, 3] for the 4-state protocol (BB84) and in [4.5] for the 6-state protocol (note that for the latter $\left.\left\langle\phi_{\uparrow} \mid \phi_{\downarrow}\right\rangle=0\right)$. This provides the joint probability distribution $P_{X Y Z}$ of the random variables $X, Y$, and $Z$ to which Alice, Bob, and Eve have access, respectively. It turns out that Eve's random variable is composed of 2 bits $Z=\left[Z_{1}, Z_{2}\right]$, where $Z_{1}=X \oplus Y(\oplus=$ xor $)$, i.e., $Z_{1}$ tells Eve whether Bob received the qubit disturbed $\left(Z_{1}=1\right)$ or not $\left(Z_{1}=0\right)$ (this is a consequence of the fact that the $\psi$ and $\phi$ states in (1.2) generate orthogonal subspaces). The probability that Eve's second bit indicates the correct value of Bob's bit depends on whether the qubit was disturbed or not: $\operatorname{Prob}\left(Z_{2}=Y \mid X=Y\right)=\delta_{0}$ and $\operatorname{Prob}\left(Z_{2}=Y \mid X \neq Y\right)=\delta_{1}$ (note that for the 6-state protocol $\left.\delta_{1}=1\right)$. The relevant Shannon informations are then:

$$
\begin{aligned}
\mathcal{I}_{\text {Bob }} & =1+\mathcal{F} \log _{2}(\mathcal{F})+(1-\mathcal{F}) \log _{2}(1-\mathcal{F}) \\
\mathcal{I}_{\text {Eve }} & =\mathcal{F}\left(1+\delta_{0} \log _{2}\left(\delta_{0}\right)+\left(1-\delta_{0}\right) \log _{2}\left(1-\delta_{0}\right)\right) \\
& +(1-\mathcal{F})\left(1+\delta_{1} \log _{2}\left(\delta_{1}\right)+\left(1-\delta_{1}\right) \log _{2}\left(1-\delta_{1}\right)\right)
\end{aligned}
$$

For the 4-state protocol Eve's Shannon information is maximal when $\delta_{0}=\delta_{1}=\frac{1}{2}+\sqrt{\mathcal{F}(1-\mathcal{F})}$. Let us concentrate on the point where $\mathcal{I}_{B o b}=\mathcal{I}_{\text {Eve }}$, at $Q B E R_{0}=$ $1-\mathcal{F}_{0}=\frac{1-1 / \sqrt{2}}{2}$. For QBERs below this threshold, Bob has more information than Eve, while above $\mathrm{QBER}_{0} \mathrm{Bob}$ has less information than Eve. We shall see that in the latter case Alice and Bob can still exploit their authenticated classical communication channel to overcome their initial drawback. Note that the noise corresponding to $\mathrm{QBER}_{0}$ in the 4-state protocol is precisely the limit above which Bell inequality [6] can no longer be violated [7,2,3], while for the 6-state protocol it corresponds to the optimal Universal Quantum Cloning Machine [8].

The situation that results when the 4-state or the 6state protocol is used, and if all the parties obtain classical random variables by carrying out measurements after each bit sent, is a special case of the more general scenario of secret-key agreement by public discussion from common information described by Maurer [9]. In this setting, two parties Alice and Bob who are willing to generate a secret key have access to repeated independent realizations of (classical) random variables $X$ and $Y$, respectively, whereas an adversary Eve learns the outcomes of a random variable $Z$. Let $P_{X Y Z}$ be the joint distribution of the three random variables. Additionally, Alice and Bob are allowed to communicate over a noiseless and authenticated, but otherwise completely insecure channel. In this situation, the secret-key rate $S(X ; Y \| Z)$ has been defined as the maximal rate at which Alice and Bob can generate a secret key that is equal for Alice and Bob with overwhelming probability and about which Eve has only a negligible amount of information (in terms of Shannon entropy). For a detailed description of the general scenario and the secret-key rate as well as for various bounds on $S(X ; Y \| Z)$, see 9911 .

A first result analyses the case when Bob's random variable $Y$ provides more (Shannon-) information about Alice's $X$ than Eve's $Z$ does (or vice versa): then this advantage can be exploited to generate a secret key:

$$
\begin{aligned}
S(X ; Y \| Z) & \geq \\
\max & \{I(X ; Y)-I(X ; Z), I(Y ; X)-I(Y ; Z)\}
\end{aligned}
$$

This bound follows from an earlier result by Csiszár and Körner [12]. It guarantees the possibility of secret-key agreement using error correction and (classical) privacy amplification whenever Bob has more information on Alice's bits than Eve. It is somewhat surprising that this bound is not tight, in particular that secret-key agreement is even be possible when the right-hand side of (6) vanishes or is negative. However, it was shown that the positivity of the expression on the right-hand side of (6) is a necessary condition for the possibility of secret-key agreement by one-way communication: whenever Alice and Bob start in a disadvantageous situation compared to Eve, then feedback is necessary. The corresponding initial phase of the key-agreement protocol is then called advantage distillation.

Let us come back to quantum cryptography and first briefly apply the general scenario for secret-key agreement to the case when Bob has more information than Eve. This case is relatively simple since, before starting the classical phase of the protocol, Bob has already an advantage over Eve. Hence inequality (6) guarantees that a positive secret-key rate can be achieved using only error correction and privacy amplification. Actually, inequality (6) provides even a lower bound on the achievable secret-bit rate.

Next, we consider the case when Bob has less information than Eve. This case may seem hopeless. However, there are still two reasons to keep optimism. First, we shall see that for QBERs not too large Alice's and Bob's qubits are still entangled, hence the technique of Quantum Privacy Amplification (QPA) [13] can be used. Next, Alice and Bob can take advantage of the authenticity of the public channel and carry out an advantage distillation protocol. We shall successively analyze these two possibilities and show that despite their differences, the former processing quantum information while the second is entirely classical, both can be applied up to precisely the same maximum $\mathrm{QBER}_{\max }$ !

The qubit sent by Alice to Bob is initially maximally entangled with another qubit that stays in Alice's hands. Once Bob received his qubit, Alice and Bob qubits are in a mixed state $\rho_{A B}$. If $\rho_{A B}$ is separable, then the cor- 
relation between Alice and Bob could be established by purely local operations and classical (public) discussions, hence there is no way for Alice and Bob to base a secret key on this correlation. If, however, $\rho_{A B}$ is entangled, then it contains some quantum (i.e. non-classical) correlations. If Alice and Bob have many pairs of qubits, each in an entangled state $\rho_{A B}$, they can apply Quantum Privacy Amplification (also called entanglement distillation or purification) 13]: after some local action involving pairs of qubit pairs and some public discussion, some qubits pairs will be more entangled at the cost that some other qubit pairs are destroyed. Repeated use of this protocol provides Alice and Bob with qubit pairs arbitrarily close to maximal entanglement, as would have been obtained with a perfect channel. They can thus be used for secret-key agreement.

According to the general incoherent eavesdropping strategy (1,2) and assuming Alice prepares the qubits in the singlet state, $\rho_{A B}$ takes the form (in the computational basis $\{|00\rangle,|01\rangle,|10\rangle,|11\rangle\})$ :

$$
\rho_{A B}=\frac{1}{2}\left(\begin{array}{cccc}
\mathcal{D} & 0 & 0 & -\mathcal{D} c_{\phi} \\
0 & \mathcal{F} & -\mathcal{F} c_{\psi} & 0 \\
0 & -\mathcal{F} c_{\psi} & \mathcal{F} & 0 \\
-\mathcal{D} c_{\phi} & 0 & 0 & \mathcal{D}
\end{array}\right)
$$

where $c_{\psi}=\left\langle\psi_{\uparrow} \mid \psi_{\downarrow}\right\rangle$ and $c_{\phi}=\left\langle\phi_{\uparrow} \mid \phi_{\downarrow}\right\rangle$. Using the PeresHorodecki 14 separability condition, one finds that $\rho_{A B}$ is entangled if and only if $\mathcal{D}>\mathcal{F} c_{\psi}$ and $\mathcal{F}>\mathcal{D} c_{\phi}$. Using the explicit relations among $\mathcal{F}, \mathcal{D}, c_{\psi}$ and $c_{\phi}$ one obtains the maximum $\mathrm{QBER}_{\max }$ for which $\rho_{A B}$ is entangled and thus QPA can be applied:

$$
\begin{aligned}
& \text { for the } 4 \text {-state protocol }: Q B E R_{\max }=1 / 4 \\
& \text { for the } 6 \text {-state protocol }: Q B E R_{\max }=1 / 3
\end{aligned}
$$

Note that these bounds are not only valid for QPA, but more generally for any quantum algorithm. Indeed, if the above bounds are violated, then Alice and Bob do not share any entanglement, hence their correlation could be produced by public discussions.

We now discuss the case when Alice, Bob and Eve are left with classical random variables $X, Y$ and $Z$, respectively, and the mutual information Bob-Alice is lower than the Eve-Alice one. Alice and Bob can then not achieve a secret key using only error correction and privacy amplification. Nevertheless, they can try to use an advantage-distillation protocol. The idea is that Alice uses her random variable $X$ to send over the public channel an $N$-bit block encoding a single bit $C$ [15]:

$$
X^{N} \oplus C^{N}:=\left[X_{1} \oplus C, X_{2} \oplus C, \ldots, X_{N} \oplus C\right]
$$

Bob then computes $\left(X^{N} \oplus C^{N}\right) \oplus Y^{N}$ and (publicly) accepts exactly if this block is equal to either $[0,0, \ldots, 0]$ or $[1,1, \ldots, 1]$, corresponding to $C=0$ and $C=1$, respectively. In other words, Alice and Bob make use of a repeat code of length $N$ with only two codewords $[0,0, \ldots, 0]$ and $[1,1, \ldots, 1]$. In this way the probability that Bob accepts erroneously a bit $C$ goes down like $\mathcal{D}^{N}$. Eve, on her side, has to use a majority vote to guess the bit $C$. Hence Bob's information on $C$ might be larger than Eve's information even in cases where Bob's information on $X^{N}$ is lower than Eve's. The following theorem defines the possibility for Alice and Bob to achieve secret-key agreement using classical algorithms:

Theorem 1 Secret-key agreement is possible if and only if

$$
\frac{\mathcal{D}}{1-\mathcal{D}}<2 \sqrt{\left(1-\delta_{0}\right) \delta_{0}}
$$

holds. When applied to the 4- and 6-state quantum cryp-

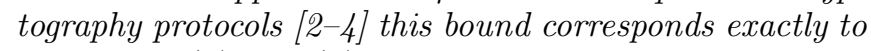
conditions (8) and (9), respectively.

Recall that $\delta_{0}$ is the probability that Eve guesses correctly the value of a bit received undisturbed by Bob.

Proof of Theorem 1: First, we note that the condition (11) is clearly necessary. Indeed, for a disturbance $\mathcal{D}$ larger than this bound the correlations between Alice and Bob correspond to a separable state $\rho_{A B}$ which can be produced without any quantum channel, simply by public discussion.

Next, we prove that the condition (11) is also sufficient. When applying the advantage distillation protocol described above, Bob's conditional error probability $\beta_{N}$ when guessing the bit sent by Alice, given that he accepts, is

$$
\beta_{N}=\frac{1}{p_{a, N}} \cdot \mathcal{D}^{N} \leq\left(\frac{\mathcal{D}}{1-\mathcal{D}}\right)^{N}
$$

where $p_{a, N}=\mathcal{D}^{N}+(1-\mathcal{D})^{N}$ is the probability that Bob accepts the received block. It is obvious that Eve's optimal strategy for guessing $C$ is to compute the block $\left[\left(C \oplus X_{1}\right) \oplus Z_{1}, \ldots,\left(C \oplus X_{N}\right) \oplus Z_{N}\right]$ and guess $C$ as 0 if at least half of the bits in this block are 0 , and as 1 otherwise. Given that Bob correctly accepts, Eve's error probability when guessing the bit $C$ with the optimal strategy is lower bounded by $1 / 2$ times the probability that she decodes to a block with $N / 2$ bits 0 and the same number of 1's. Hence we get that

$\gamma_{N} \geq \frac{1}{2}\left(\begin{array}{c}N \\ N / 2\end{array}\right)\left(1-\delta_{0}\right)^{N / 2} \delta_{0}^{N / 2} \geq \frac{k}{\sqrt{N}} \cdot\left(2 \sqrt{\left(1-\delta_{0}\right) \delta_{0}}\right)^{N}$

holds for some constant $k$ and for sufficiently large $N$ by using Stirling's formula. Note that Eve's error probability given that Bob accepts is asymptotically equal to her error probability given that Bob correctly accepts because Bob accepts erroneously only with asymptotically vanishing probability, given that he accepts.

Although it is not the adversary's ultimate goal to guess the bits $C$ sent by Alice, it has been shown that the fact that $\beta_{N}$ decreases exponentially faster than 
$\gamma_{N}$ implies that for sufficiently large $N$, Bob has more (Shannon-) information about the bit $C$ than Eve (see for example [11], [10]). Hence Alice and Bob have managed to generate new random variables with the property that Bob obtains more information about Alice's random bit than Eve has. Thus $S(X ; Y \| Z)>0$ holds for this, hence also for the original, situation because of the bound (6).

In conclusion, we have proven that secret-key agreement using either 4-state or 6-state quantum cryptography, assuming general incoherent eavesdropping, is possible if and only if the quantum bit error rate is lower than that produced by the intercept-resend eavesdropping strategy (1/4 and $1 / 3$ for the 4 -state and 6 -state protocols, respectively). This limit corresponds to complete disentanglement and is valid as well if Alice and Bob use quantum information processing, like quantum privacy amplification, as if they use classical information processing, like advantage distillation [16]. In other words, as long as Alice and Bob share some entanglement, they can use either a quantum protocol or a classical protocol to extract a secret-key from this entanglement.

Quantum Privacy Amplification uses quantum controlled-not gates, the basic building block of quantum computers. The latter are usually thought as fundamentally more efficient than classical computers. In the case of quantum cryptography our results demonstrate that the same task can be achieved with both types of computers, but it remains to be determined whether one method is more efficient than the other. The case of coherent eavesdropping also remains open.

From a practical point of view it is crucial to know the upper error rate compatible with secret-key agreement. It is also important to have good estimates for the secret-key rate $S(X ; Y \| Z)$. Indeed, in practice one has to optimize a compromise between high raw bit rates and low error rates [17].

Stimulating discussions with Artur Ekert, Bruno Huttner, Itoshi Inamori and Ueli Maurer are acknowledged. This work was partially supported by the Swiss National Science Foundation.

[1] C. H. Bennett and G. Brassard, in Proceedings of the IEEE International Conference on Computer, Systems, and Signal Processing, Bangalore, India (IEEE, New York, 1984), pp. 175-179; A.E. Ekert, Phys. Rev. Lett. 67, 661 (1991); see also the Physics World issue of March 1998.

[2] I. Cirac and N. Gisin, Phys. Lett. A 229, 1-7, 1997.

[3] C. Fuchs, N. Gisin, R.B. Griffiths, C.S. Niu and A. Peres, Phys. Rev. A 56, 1163, 1997.
[4] H. Bechmann-Pasquinucci and N. Gisin, quant-ph 9807041 .

[5] D. Bruss, Phys. Rev. Lett. 81, 3018, 1998.

[6] J.F. Clauser, M.A. Horne, A. Shimony and R.A. Holt, Phys. Rev. Lett. 23, 880, 1969.

[7] N. Gisin and B. Huttner, Phys. Lett. A 228, 13-21, 1997.

[8] V. Bužek and M. Hillery, Phys. Rev. A 54, 1844 (1996); N. Gisin and S. Massar, Phys. Rev. Lett. 79, 2153-2156, 1997.

[9] U. M. Maurer, Secret key agreement by public discussion from common information, IEEE Transactions on Information Theory, Vol. 39, No. 3, pp. 733-742, 1993.

[10] U. M. Maurer and S. Wolf, Unconditionally secure key agreement and the intrinsic conditional information, to appear in IEEE Transactions on Information Theory, 1999.

[11] U. M. Maurer and S. Wolf, Unconditionally secure secretkey agreement and the intrinsic conditional mutual information, Tech. Rep. 268, Department of Computer Science, ETH Zürich, May 1997.

[12] I. Csiszár and J. Körner, Broadcast channels with confidential messages, IEEE Transactions on Information Theory, Vol. IT-24, pp. 339-348, 1978.

ref. therein;

[13] D. Deutsch et al., Phys. Rev. Lett. 77, 2818, 1996; Ch. H. Bennett et al., Phys. Rev. Lett. 76, 722, 1996.

[14] A. Peres, Phys. Rev. Lett. 77, 1413, 1996; M., R. \& P. Horodecki, Phys. Lett. A 223, 1, 1996.

[15] Note that there exist much more efficient protocols in terms of the amount of extractable secret key. However, since we only want to prove a qualitative possibility result here, it is sufficient to look at this simpler protocol.

[16] Note that when the disturbance caused by Eve is so low that she has less (Shannon) information than Bob on the bits encoded in individual qubits, then neither quantum privacy amplification nor advantage distillation is needed, classical error correction and privacy amplification suffice. Hence, the terminology quantum privacy amplification is unfortunate, as it does not correspond to classical privacy amplification, but include advantage distillation.

[17] H. Zbinden et al., Applied Physics B 67, 743-748, 1998, and ref. therein; G. Ribordy et al., Electron. Lett. 34, 2116-2117, 1998. 


\section{FIGURE CAPTIONS}

Fig. 1: Eve and Bob information versus the QBER, here plotted for incoherent eavesdropping on the 4-state protocol. For QBERs below $\mathrm{QBER}_{0}$, Bob has more information than Eve and secret-key agreement can be achieved using classical error correction and privacy amplification. These can, in principle, be implemented using only 1-way communication. The secret-key rate can be at least as large as the information differences. For QBERs above $\mathrm{QBER}_{0}$, Bob has a disadvantages with respect to Eve. Nevertheless, Alice and Bob can apply quantum privacy amplification up to the QBER corresponding to the intercept-resend eavesdropping strategies, $\mathrm{IR}_{4}$ and $\mathrm{IR}_{6}$ for the 4 -state and 6-state protocols, respectively. Alternatively, they can apply a classical protocol called advantage distillation which is effective precisely up to the same maximal QBER $\mathrm{IR}_{4}$ and $\mathrm{IR}_{6}$. Both the quantum and the classical protocols require then 2-way communication. Note that for the eavesdropping strategy optimal from Eve' Shannon point of view on the 4-state protocol, $\mathrm{QBER}_{0}$ correspond precisely to the noise threshold above which Bell inequality can no longer be violated. For the 6 -state protocol a similar relation with optimal universal quantum cloning holds [8]. 


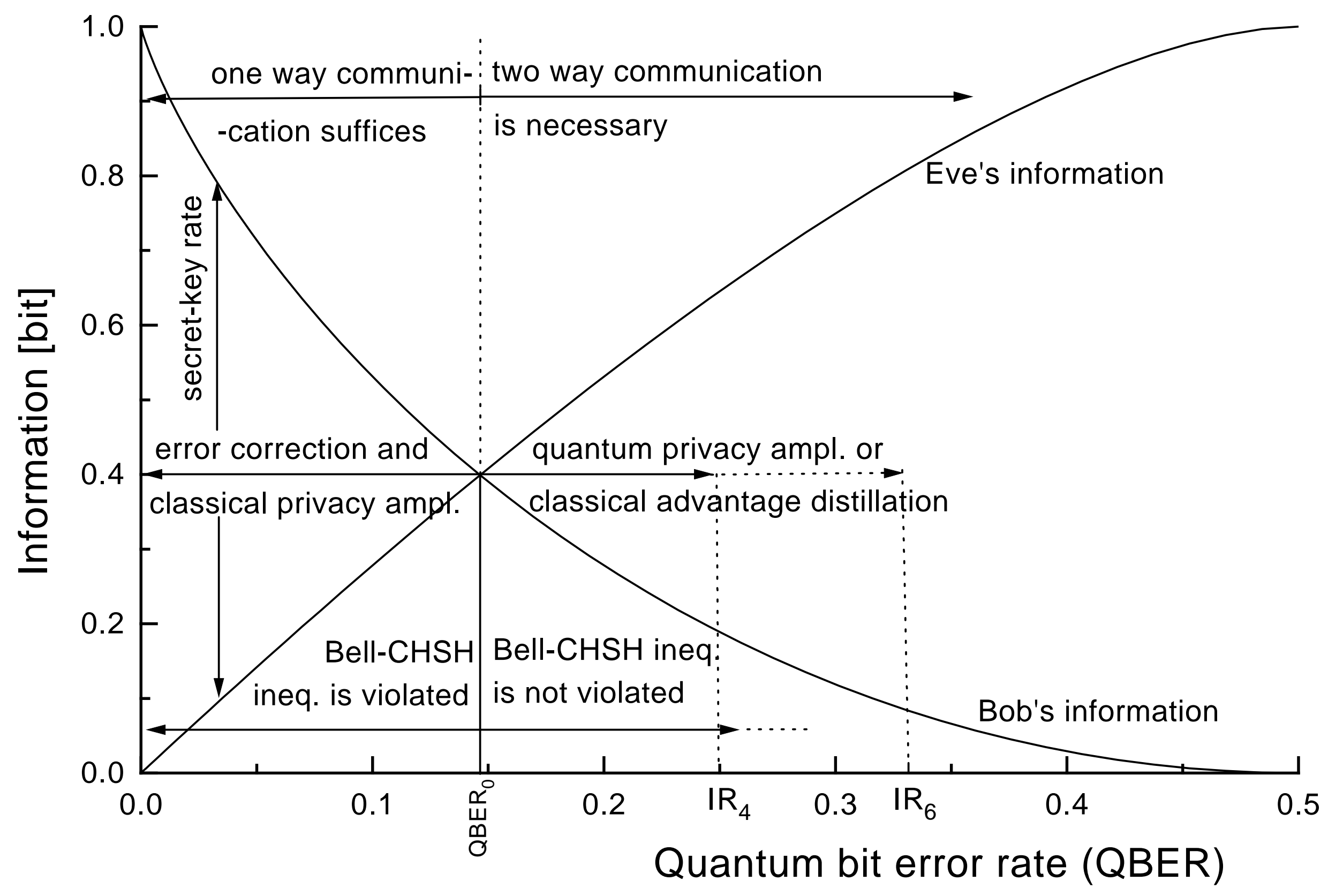

